ABHANDLUNGEN DER DEUTSCHEN AKADEMIE DER WISSENSCHAFTEN Z U B ERLIN

Klasse für Mathematik, Physik und Technik

Jahrgang 1961 Nr. 1

ERICH KÄHLER

\title{
DIE DIRAC-GLEICHUNG
}

A K A D E M IE - VERLAG - B ERLIN

1961 
Vorgelegt in der Gesamtsitzung vom 16. Juni 1960

Zum Druck genehmigt am gleichen Tage, ausgegeben am 31. März 1961.

\author{
Erschienen im Akademie-Verlag GmbH, Berlin W 8, Leipziger StraBe 3-4 \\ Lizenz-Nr. 202 100/501/61 \\ Copyright 1961 by Akademie-Verlag GmbH, Berlin \\ Alle Rechte vorbehaiten \\ Gesamtherstellung: VEB Druckerei „Thomas Müntzer" Bad Langensalza \\ Bestellaummer: 2001/61/1/1 \\ Preis: DM 5,60 \\ Printed in Germany \\ ES 19 B 4
}


Herrn Werner Schmeidler, Berlin, zum 70. Geburtstage gewidmet 

Ob der innere Differentialkalkül der Quantentheorie echten Dienst zu leisten vermag, muß sich zunächst an der Gleichung zeigen, aus deren Beobachtung er entstanden ist, an der Dirac-Gleichung für die Zustände des Elektrons im elektromagnetischen Felde.

Bei dem großen Erfolge der DrRacschen Theorie kann von einer neuen Auffassung der DiRAc-Gleichung kaum mehr erwartet werden als die Bestätigung bekannter Ergebnisse, aber vielleicht verdienen manche Züge dieser durch den Kalkül nahezu eindeutig vorgeschriebenen Behandlung der DrRacschen Theorie auch vom physikalischen Standpunkte nähere Betrachtung.

Für diesen Fall sind in der vorliegenden Abhandlung einige Rechnungen mitgeteilt, die einen Vergleich mit der üblichen Auffassung der Drracschen Theorie ermöglichen.

Um die in einer vorangegangenen Arbeit ${ }^{1}$ ) entwickelten Grundlagen des inneren Differentialkalküls bequem zitieren zu können, setzt die Numerierung der Abschnitte diejenige aus jener Arbeit einfach fort, und auch die Formelzitate weisen, wenn sie durch Ziffern unter 100 geschehen, auf Formeln der früheren Arbeit.

Eine Inhaltsangabe erübrigt sich durch die Bemerkung, daß die Überschriften zu den einzelnen Abschnitten den Gang der Untersuchung hinreichend kennzeichnen.

36.

\section{Zustandsdifferentiale}

Der Drracschen Theorie des Elektrons liegt wie der Maxwelischen Theorie die ErNSTEIN-MinkowsKI-Metrik

$$
\left(d x^{1}\right)^{2}+\left(d x^{2}\right)^{2}+\left(d x^{3}\right)^{2}-c^{2} d t^{2}
$$

zugrunde.

In dem auf diese Metrik bezogenen inneren Differentialkalkül nimmt die DIRAc-Gleichung des Elektrons im elektromagnetischen Felde die Gestalt

an, wo

$$
\frac{h c}{2 \pi i} \delta u=\left(i E_{0}+e \omega\right) \vee u
$$

das durch

$$
\omega=A_{1} d x^{1}+A_{2} d x^{2}+A_{3} d x^{3}-c V d t
$$

$$
d \omega=H_{1} d x^{2} \wedge d x^{3}+H_{2} d x^{3} \wedge d x^{1}+H_{3} d x^{1} \wedge d x^{2}+c\left(E_{1} d x^{1}+E_{2} d x^{2}+E_{3} d x^{3}\right) \wedge d t
$$

bis auf ein additives totales Differential bestimmte elektromagnetische Differential ist.

1) ERIch KXHLER, Innerer und äußerer Differentialkalkül, Abh. der Deutschen Akademie der Wissenschaften zu Berlin, Kl. für Math., Phys. und Technik, 1960 Nr. 4. 